\title{
Croyances de survie et choix de contrat d'assurance décès : une étude empirique
}

\author{
Meglena Jeleva* \\ LEN-C3E, Université de Nantes et EUREQua
}

\section{Introduction}

Les croyances jouent un rôle fondamental dans les décisions des individus en présence d'incertitude car, en déterminant la vraisemblance perçue des événements futurs, elles interviennent dans l'évaluation de la satisfaction correspondant à chacune des décisions possibles. Ces croyances sont le résultat de l'information objective dont disposent les individus et aussi de leur attitude vis-à-vis de l'incertitude. Une littérature théorique foisonnante s'est intéressée aux choix des individus en présence d'incertitude analysant aussi bien les situations dans lesquelles les individus connaissent parfaitement la distribution de probabilités des états de la nature (situation de risque) que les situations d'incertitude non-probabilisée où l'information est incomplète ${ }^{1}$. L'élaboration de ces modèles théoriques a été accompagnée par un ensemble tout aussi riche d'études expérimentales visant à tester aussi bien les axiomes que les représentations des préférences des différents modèles théoriques proposés.

En revanche, la manière de laquelle les individus forment leurs croyances à partir des informations objectives à leur disposition et le lien entre ces croyances et les choix effectifs a été peu exploré à cause de la quantité importante d'information nécessaire pour ce type d'études.

Les croyances de survie sont les rares à avoir fait l'objet de plusieurs études, probablement à cause de leur impact sur des décisions qui présentent un intérêt fondamental pour les économistes. Des enquêtes concernant

\footnotetext{
* Je remercie P. A. Chiappori, M. Visser et deux rapporteurs pour leurs commentaires et suggestions.

1 Pour des revues de la littérature sur ces modèles, voir Willinger (1990) et Cohen. Tallon (2000).
} 
ce type de croyances ont été menées, entre autres, par Lichtenstein et alii (1978), Hamermesh (1985), Benjamin, Dougan (1997). Ces travaux étudient les biais de perceptions des risques liés à différentes causes. Il apparaît que les individus ont tendance à surestimer les risques associés à des événements rares (et respectivement à sous-estimer les risques liés à des événements plus fréquents). Par ailleurs, l'information disponible sur l'état de santé et sur les causes statistiques de décès semble utilisée de manière rationnelle.

Ces études laissent cependant ouverte la question de l'impact des croyances de survie sur les décisions réelles et ne comportent que peu d'éléments permettant d'établir un lien entre ces croyances, l'information objective dont dispose l'individu et son attitude vis-à-vis de l'incertitude.

L'objectif principal de cet article est d'abord d'étudier la formation des croyances de survie et ensuite, d'établir un lien entre croyances de survie et décisions en matière de contrat d'assurance décès ${ }^{2}$ en testant des prédictions des modèles théoriques concernant la demande d'assurance. Notons que l'assurance décès est un type particulier de contrat d'assurance, dans la mesure où l'indemnité, (ou, pour utiliser la terminologie spécifique à ce type de contrats, le capital assuré), ne revient pas à la personne assurée, mais à ses héritiers. Il en découle que des caractéristiques supplémentaires comme l'altruisme, peuvent être déterminantes dans le fait d'acheter un tel contrat.

L'étude de la formation des croyances et de leur impact sur les choix d'assurance décès nous fournit aussi l'occasion de nous interroger, plus généralement, sur les déterminants des choix de contrats d'assurance décès.

Les données utilisées proviennent d'une étude sur l'état de santé et le système de retraites d'américains approchant de l'âge de la retraite ou déjà retraités, menée en 1992 par l'Université de Michigan pour le compte de l'Institut National de la Vieillesse et de la Santé : Health and Retirement Study (HRS).

Les variables que nous utilisons dans notre étude peuvent être regroupées en trois catégories:

- variables relatives aux contrats d'assurance décès : possession ou non d'un contrat, prime annuelle et capital assuré;

- variables relatives à la survie : croyances, âge, sexe, caractéristiques de l'état de santé (se traduisant par l'existence ou non de certaines maladies), tabagisme;

- variables pouvant avoir un effet sur le contrat d'assurance choisi : statut matrimonial, nombre d'enfants, revenu.

Les principaux résultats de cette étude sont les suivants. L'étude de l'impact des caractéristiques de santé sur les croyances met en évidence la

2 Un contrat d’assurance décès garantit, en cas de décès du souscripteur pendant une période donnée après la signature du contrat, le paiement à ses héritiers d'un capital spécifié dans le contrat. 
rationalité, en moyenne, des personnes interrogées. Il apparaît clairement que les maladies pouvant avoir des conséquences fatales, et le fait de fumer, réduisent les croyances de survie : parmi les maladies, celles qui s'avèrent les plus significatives dans la formation des croyances sont les maladies cardiovasculaires, suivies du diabète et des maladies gastro-entérologiques. On observe, par ailleurs, que les maladies ne sont pas suffisantes pour expliquer les croyances et que, une fois leur effet isolé, il reste un important élément subjectif, qui peut être lié à l'attitude vis-à-vis de l'incertitude.

Les résultats concernant la décision d'achat ou non d'un contrat d'assurance et le montant de capital assuré laissent penser que la décision d'achat d'un contrat est antérieure au choix du montant assuré. En effet, si l'achat ou non d'un contrat peut être expliqué par la présence d'héritiers et le revenu, le capital assuré est lui, lié aux croyances de survie (dans leur partie objective et subjective) et à l'âge. On a ainsi une confirmation du fait que, toutes choses égales par ailleurs, l'attitude vis-à-vis de l'incertitude, qui transite ici par la partie subjective des croyances a bien un effet positif sur le capital assuré. On constate de plus que le revenu a un effet positif aussi bien sur la décision d'acheter un contrat que sur le montant de capital assuré ce qui montre que l'assurance est un bien normal.

La section suivante de l'article présente un modèle simple de choix d'assurance décès, faisant intervenir les croyances des assurés. Nous décrivons ensuite la base de données et donnons les statistiques descriptives des principales variables utilisées. La section 4 est consacrée à l'analyse des croyances de survie et à leur décomposition en croyances objectives et subjectives. La dernière section de l'article est consacrée aux déterminants de la demande d'assurance décès.

\section{La demande d'assurance décès : quelques résultats théoriques dans le cadre d'un modèle simple}

Les contrats d'assurance décès, comme les contrats d'assurance vie, sont des contrats de type particulier : $\mathrm{si}$, comme tous les contrats d'assurance, ils font intervenir des transferts de richesse entre différents états de la nature, ils font en outre intervenir des transferts entre différentes générations. Ces décisions sont souvent prises simultanément avec des décisions d'épargne qui, elles, font intervenir des transferts entre différentes périodes de la vie. Ce sont ces facteurs qui expliquent le fait que les analyses de ces décisions sont faites la plupart du temps, dans le cadre de modèles dynamiques de «cycle de vie ». On peut citer dans ce cadre, entre autres, les études de Yaari (1965) et Fisher (1973).

Nous nous intéressons dans cette section plus particulièrement à l'impact du risque de décès et des croyances de survie ainsi que de l'altruisme, sur les décisions d'achat d'assurance décès. 
L'objectif de ce modèle très simple, qui s'inspire de Henriet-Rochet (1992), est de mettre en évidence certains résultats de statique comparative qui pourront être testés dans l'étude empirique qui constitue l'objectif principal de cet article.

Soit $w$ le revenu qu'un agent a décidé d'allouer à la deuxième période de sa vie, sachant qu'il peut vivre ou non pendant cette deuxième période.

L'agent a deux possibilités pour le revenu transféré vers la deuxième période : l'épargner, auquel cas il se transformera en consommation pour lui s'il est en vie ou reviendra à ses héritiers sinon; ou bien acheter de l'assurance décès, auquel cas les seuls qui peuvent en bénéficier sont ses héritiers, s'il décède.

On note $\theta$ et $\bar{\theta}$ les deux états de la nature possibles en deuxième période, avec $\theta=$ ll'agent est en vie $\}$ et $\bar{\theta}=$ \{l'agent n'est pas en vie $\}. W$ est le revenu aléatoire en deuxième période. Si on note $E$ l'épargne, dont le taux d'intérêt est $r$, et $C$ le capital décès acheté, on a :

$$
W(\theta)=(1+r) E \text { et } W(\bar{\theta})=(1+r) E+C .
$$

Les préférences de l'individu sont représentées par le modèle d'espérance d'utilité.

Dans la représentation des préférences de l'individu interviennent :

- une fonction d'utilité $u(\cdot)$, supposée concave;

- une probabilité subjective de décès $q$, qui sera par la suite appelée « croyance »;

- un paramètre d'altruisme $k \in[0,1]$;

- le prix par franc de capital décès $p$.

Le paramètre d'altruisme est une pondération de l'utilité dans l'état de la nature où l'agent n'est pas en vie. Lorsque $k=0$, l'agent ne tient pas compte dans ses décisions de l'utilité de ses héritiers et si $k=1$ leur richesse a autant de poids dans ses décisions que la sienne propre.

Le montant épargné et le capital décès acheté par cet agent sont solutions du programme suivant :

$$
\begin{gathered}
\max _{E, C}\{q k u[(1+r) E+C]+(1-q) u[(1+r) E]\} \\
\text { sous la contrainte } \\
w=E+p C
\end{gathered}
$$

En exprimant $E$ à partir de $w$ et $C$, on réduit le nombre de variables et on obtient :

$$
\max _{C \in\left[0, \frac{w}{p}\right]}\{q k u[(1+r)(w-p C)+C]+(1-q) u[(1+r)(w-p C)]\}
$$

On note $V($.$) la fonction objectif du programme ci-dessus.$ 
La concavité de la fonction d'utilité $u(\cdot)$ implique que la condition de second ordre du programme ci-dessus est vérifiée.

La condition de premier ordre s'écrit :

$$
\begin{aligned}
& q(1-(1+r) p) k u^{\prime}[(1+r)(w-p C)+C]- \\
& (1-q)[(1+r) p] u^{\prime}[(1+r)(w-p C)] \leqslant 0
\end{aligned}
$$

On note :

$$
\begin{gathered}
V^{*}(C, p, q, \ldots)=q(1-(1+r) p) k u^{\prime}[(1+r)(w-p C)+C]- \\
(1-q)[(1+r) p] u^{\prime}[(1+r)(w-p C)]
\end{gathered}
$$

Ainsi, en notant $C^{*}$, le montant optimal de capital décès, solution de (1), on a

$$
C^{*}=0 \text { si } V^{*}(0, p, q, \ldots) \leqslant 0
$$

sinon,

$$
C^{*}>0 \text { et vérifie } V^{*}\left(C^{*}, p, q, \ldots\right)=0 .
$$

On suppose, dans ce qui suit, que

$$
(1-(1+r) p)>0 \text {. }
$$

Si cette condition n'est pas vérifiée, $V^{*}(0, p, q, \ldots)<0$ quelles que soient les valeurs des autres paramètres du modèle et donc l'agent n'achète pas d'assurance décès.

La proposition suivante regroupe des résultats de statique comparative concernant l'impact de l'altruisme et de la richesse sur le capital assuré. Ces résultats sont standard (cf. par exemple Henriet, Rochet 1992 ou Eeckhoudt, Gollier 1992) et leur preuve est immédiate.

Proposition 1 L'impact de l'altruisme et de la richesse sur le montant de capital assuré est le suivant:

(i) si l'agent n'est pas altruiste $(k=0)$, il n'achète pas d'assurance décès;

(ii) si l'agent est altruiste $(k>0)$, le capital assuré augmente avec l'altruisme;

(iii) si le coefficient absolu d'aversion pour le risque de l'agent est décroissant, le capital assuré crô̂t avec la richesse.

Les deux premiers résultats de la proposition sont très intutifs. Si un agent n'a pas d'héritiers ou ne s'en préoccupe pas, il n'a aucune raison d'acheter de l'assurance décès. Le troisième résultat montre que l'assurance décès est un bien normal sous l'hypothèse assez courante de décroissance de l'aversion absolue pour le risque. Ainsi, si l'agent devient moins averse au risque quand sa richesse augmente, il achètera plus d'assurance décès. Ce résulat peut paraître surprenant dans la mesure où lorsqu'on étudie la 
demande d'assurance dommage, on obtient le résultat inverse. On comprend bien ce phénomène car, dans le cas standard, réduire le risque au sens de Rothschild et Stiglitz consiste à acheter plus d'assurance, alors que dans le cas de l'assurance décès, on réduit le risque au sens classique, en réduisant le montant d'assurance décès. Ainsi, lorsque l'on devient moins averse au risque avec la richesse, on va acheter plus d'assurance décès.

On s'intéresse maintenant à l'impact de la prime unitaire $p$ et de la croyance de décès $q$ sur le capital assuré. Deux cas seront considérés. On étudie dans un premier temps le cas où prime et croyance sont indépendantes. Ceci peut correspondre à une situation où les croyances des individus sont purement subjectives et ne dépendent pas d'informations objectives, alors que la prime d'assurance, calculée à partir d'informations statistiques, s'appuie sur des informations objectives. Dans un second temps, on suppose que ces deux paramètres sont tous les deux liés à la probabilité objective de décès.

Proposition 2 Si la prime unitaire est indépendante de la croyance de survie des agents, alors:

(i) la capital décès croît avec la croyance de décès;

(ii) si l'aversion relative pour le risque est inférieure à 1 , alors le capital décès décroît avec la prime par franc de capital décès.

\section{Preuve}

(i) Si $p$ et $q$ sont indépendants, on obtient :

$$
\frac{d C^{*}}{d q}=-\frac{k(1-(1+r) p) u^{\prime}[(1+r)(w-p C)+C]+[(1+r) p] u^{\prime}[(1+r)(w-p C)]}{\frac{\partial^{*}}{\partial C}}>0 .
$$

(ii) Si $p$ et $q$ sont indépendants,

$$
\frac{d C^{*}}{d p}=-\frac{-q(1+r) k u^{\prime}(A)-q(1-(1+r) p) C k(1+r) u^{\prime \prime}(A)-(1-q)(1+r) u^{\prime}(B)+(1-q)(1+r)^{2} p C u^{\prime \prime}(B)}{\frac{\partial V^{*}}{\partial C}}
$$

Le signe de $\frac{d C^{*}}{d p}$ est déterminé par le signe de $D_{2}$ avec

$$
\begin{aligned}
D_{2}= & -q(1+r) k u^{\prime}(A)-q(1-(1+r) p) C k(1+r) u^{\prime \prime}(A)- \\
& -(1-q)(1+r) u^{\prime}(B)+(1-q)(1+r)^{2} p C u^{\prime \prime}(B)
\end{aligned}
$$

Après transformations, $D_{2}$ s'écrit :

$$
\begin{aligned}
D_{2}= & q(1+r) k u^{\prime}(A)\left[-1-\frac{A u^{\prime \prime}(A)}{u^{\prime}(A)}\right]+(1-q)(1+r) u^{\prime}(B)\left[-1-\frac{B u^{\prime \prime}(B)}{u^{\prime}(B)}\right] \\
& +(1+r)^{2} w\left[q k u^{\prime \prime}(A)+(1-q) u^{\prime \prime}(B)\right]
\end{aligned}
$$

et donc, si $u$ est telle que $-\frac{x u^{\prime \prime}(x)}{u^{\prime}(x)}<1, \forall x$, alors $D_{2}<0$ et donc $\frac{d C^{*}}{d p}<0$. 
Les résultats de la proposition précédente sont intuitifs et en accord avec les résultats classiques sur la variation de la demande d'assurance en fonction des croyances et du prix (cf. Eeckhoudt, Gollier 1992 ou Schlesinger 2000). Comme dans le cas standard, à moins d'imposer une restriction sur l'aversion relative pour le risque, l'assurance décès peut être un bien de Giffen.

Considérons maintenant le cas où la croyance de décès et le prix unitaire de l'assurance décès dépendent tous les deux de la probabilité objective de décès. On note cette probabilité $\tilde{p}$. Cette hypothèse est justifiée si on suppose que les agents sont rationnels et informés sur les caractéristiques qui ont une influence sur le risque de décès et si on tient compte du fait que les assureurs font des estimations et proposent des primes liées au risque estimé de chaque individu.

La relation fonctionnelle entre les probabilités objective et subjective de décès peut prendre différentes formes en fonction du modèle de décision dans l'incertain considéré : espérance d'utilité, espérance d'utilité dépendante du rang etc $^{3}$. En toute généralité, on doit donc supposer :

$$
q=g(\tilde{p}), \text { avec } g^{\prime}>0,
$$

Afin d'obtenir des résultats théoriques plus explicites qui pourront être testés par la suite, nous adoptons une forme linéaire pour la relation entre probabilité objective et croyance ce qui correspond explicitement à un modèle avec ajustement autour d'une probabilité focale. Cette spécification conserve un degré de généralité suffisant dans notre cas particulier avec deux états de la nature.

$$
q=\tilde{p}+\varepsilon, \text { avec } \varepsilon \in R \text { et tel que } 0 \leqslant \tilde{p}+\varepsilon \leqslant 1
$$

où $\varepsilon$ est un paramètre qui mesure l'attitude vis-à-vis de l'incertitude de l'agent. $\varepsilon$ peut être considéré comme un paramètre de pessimisme car plus il est élevé, plus l'agent surestime sa probabilité de décès. Notons que $\varepsilon$ peut être négatif, ce qui correspond à une sous-estimation de la probabilité de décès.

Pour ce qui est de l'assureur, on suppose que la prime unitaire est une fonction croissante par rapport au risque objectif: $p=f(\tilde{p})$ avec $f^{\prime}>0$. On suppose de plus $f^{\prime}>1$ et $f(\tilde{p})>\tilde{p}$ ce qui permet d'introduire un chargement de la prime, correspondant aux coûts admistratifs de la compagnie d'assurance et aux chargements de sécurité généralement incorporés dans les tarifs.

Dans la proposition suivante sont regroupés quelques résultats concernant l'impact des croyances et de la probabilité objective sur le montant du capital assuré.

3 Une présentation détaillée de ces modèles et des axiomatiques correspondantes se trouve dans Willinger (1990) ot Cohen, Tallon (2000). 
Proposition 3 Si la prime unitaire et la croyance de survie des agents dépendent toutes deux de la probabilité objective de décès, alors :

(i) plus un agent est pessimiste, plus il achètera d'assurance décès;

(ii) l'impact de la probabilité objective de décès sur le montant de capital assuré est indéterminé. En particulier, si l'assurance décès est un bien Giffen, la demande d'assurance augmente avec la probabilité objective de décès.

\section{Preuve}

(i) En tenant compte du fait que $q=\tilde{p}+\varepsilon$ et en dérivant, on obtient directement,

$$
\frac{d C^{*}}{d \varepsilon}=-\frac{(1-(1+r) p) k u^{\prime}[(1+r)(w-p C)+C]+[(1+r) p] u^{\prime}[(1+r)(w-p C)]}{\frac{\partial V^{*}}{\partial C}}>0
$$

(ii) En tenant compte de la relation entre $p, q$ et $\tilde{p}$, on obtient :

$$
\frac{d C^{*}}{d \tilde{p}}=-\left(\frac{d V^{*}}{d p} f^{\prime}(\tilde{p})+\frac{d V^{*}}{d q}\right) / \frac{d V^{*}}{d C}
$$

et donc

$$
\frac{d C^{*}}{d \tilde{p}}>0 \Leftrightarrow \frac{d V^{*}}{d p} f^{\prime}(\tilde{p})>-\frac{d V^{*}}{d q}
$$

On a $\frac{d V^{*}}{d q}>0$ et donc si l'assurance décès est un bien Giffen, ce qui se traduit ici par $\frac{d V^{*}}{d p}>0$, on a $\frac{d C^{*}}{d \tilde{p}}>0$, d'après l'inégalité (9).

Ainsi, le capital assuré croît avec le paramètre de pessimisme, indépendamment de la probabilité objective. Ce résultat est intuitif, dans la mesure où le paramètre de pessimisme accroît le poids de l'événement correspondant au décès de l'individu et l'incite à augmenter le revenu affecté à cet état de la nature.

L'impact de l'accroissement du risque objectif sur le capital assuré est plus complexe à déterminer car le risque objectif influence aussi bien le coût de l'assurance (par le biais de la prime unitaire) que la croyance de décès. On constate ainsi que l'impact de la probabilité objective de décès sur le capital assuré est le résultat de l'importance relative de deux effets : un effet «prix » et un effet « accroissement du risque ». En effet, un accroissement de la probabilité objective accroît le risque, par l'accroissement de la croyance de décès et accroît le prix de l'assurance. L'impact de ces deux effets va dans le même sens quand l'assurance est un bien de Giffen. Si ce n'est pas le cas, c'est leur importance relative qui détermine l'impact de la probabilité objective sur le capital assuré.

On peut par ailleurs noter que, $\frac{d V^{*}}{d q}>0$ et $f^{\prime}>1$ impliquent $\frac{d C^{*}}{d \tilde{p}}>\frac{d C^{*}}{d p}$ et donc l'impact de la probabilité objective de décès sur la demande d'assurance est plus important que celui de la prime d'assurance. 
Ceci résulte aussi du fait qu'une variation de la probabilité objective engendre un double effet : prix et variation du risque.

On peut de la même manière tenter de déterminer ce qui, de la probabilité objective ou du paramètre de pessimisme $(\varepsilon)$, a un plus fort impact sur la demande d'assurance décès. On obtient :

$$
\operatorname{sgn}\left(\frac{d C^{*}}{d \tilde{p}}-\frac{d C^{*}}{d \varepsilon}\right)=\operatorname{sgn}\left(\frac{d V^{*}}{d p}\right)=\operatorname{sgn}\left(\frac{d C^{*}}{d p}\right)
$$

ce qui implique que la taille relative des deux effets dépend, une fois de plus, de l'effet du prix sur la demande d'assurance. Ainsi, si l'aversion relative pour le risque est inférieure à 1 , on a $\frac{d C^{*}}{d \varepsilon}>\frac{d C^{*}}{d \bar{p}}$, le pessimisme a un impact plus fort que la probabilité objective sur la demande d'assurance décès.

Dans la section suivante nous allons tester, sur des données d'enquête, les implications de ce modèle en accordant une place particulière au mécanisme de formation des croyances de décès et à leur impact sur la demande d'assurance décès.

\section{$3 \quad$ Les données}

Les données utilisées proviennent d'une étude sur l'état de santé et le système de retraites (HRS ${ }^{4}$ ) d'américains approchant de l'âge la retraite ou déjà retraités, menée par l'Université de Michigan pour le compte de l'Institut National de la Vieillesse et de la Santé et du Département Américain des Affaires Sociales. L'objectif de cette étude, réalisée en 1992, est de recueillir des informations sur les plans d'assurance retraite et d'assurance santé des citoyens américains dans la catégorie concernée, ainsi que sur leur état de santé et les facteurs déterminant leurs décisions de départ en retraite.

Ces données ont été utilisées dans plusieurs études, portant principalement sur les décisions en matière d'assurance santé (Rogowski, Karoly 2000), d'épargne (Lusardi 1998), et de choix de financement des retraites (Mitchell, Moore 1998), ainsi que dans une étude sur l'asymétrie d'information en assurance vie (Cawley, Philipson 1999).

La population cible inclut les ménages, comportant au moins un membre dans la tranche d'âge [51-61 ans]. L'échantillon contient 12652 individus de 7702 ménages. Lorsque le ménage est composé de plusieurs personnes, elles sont toutes interrogées, quel que soit leur âge. Nous ne considérons, dans notre étude, que les données relatives à la personne responsable des affaires financières du ménage.

Le questionnaire comporte des questions portant sur le statut socioéconomique, les plans de retraite, d'assurance santé, la composition du revenu, l'état de santé, l'opinion sur le système de sécurité sociale et les caractéristiques des différentes formes de contrats d'assurance-vie possédés.

4 Health and Retirement Study. 
De façon surprenante, on ne trouve pas directement certaines variables importantes, comme le statut matrimonial, le fait d'avoir des enfants etc. Il nous a fallu reconstituer ces variables par recoupements.

Les croyances des personnes interrogées sur leur probabilité de survie sont données comme réponse à la question : « En utilisant un nombre entre 0 et 10 , où 0 signifie aucune chance et 10, la certitude, dites à combien vous estimez vos chances de vivre plus de 75 ans? ".

Au États Unis, les contrats d'assurance vie, dits ordinaires, et correspondants aux contrats d'assurance décès selon la terminologie adoptée en France ${ }^{5}$, représentent $58 \%^{6}$ du volume des primes d'assurance collectées toutes branches confondues. De plus, on estime à $90 \%$ le pourcentage des ménages de deux adultes qui possèdent ce type de contrats. L'assurance vie comporte, dans la terminologie nord-américaine deux grandes catégories de contrats : les contrats d'assurance dits "vie entière », où un capital est payé aux héritiers du souscripteur, indépendamment du moment du décès et les contrats «temporaires décès », ou assurance décès à terme, selon la terminologie américaine, où un capital est versé aux héritiers du souscripteur uniquement si le décès survient pendant une période donnée. Nous ne considérons dans notre étude que ce dernier type de contrat (nous l'appellerons désormais simplement « contrat d'assurance décès ») car la prime qui lui correspond doit être liée à la probabilité de décès pendant une période donnée.

Ce contrat peut être souscrit, soit individuellement, soit par un groupe d'individus et dans certains cas, la prime de ce contrat de groupe peut être prise en charge par l'employeur de la personne possédant ce type de contrat. Parmi les individus de l'échantillon qui possèdent un contrat d'assurance décès $(50.1 \%$ de tous les individus $), 48.9 \%$ possèdent un contrat individuel, $22.2 \%$ un contrat collectif, mais dont ils payent une partie de la prime et $28.9 \%$, un contrat collectif, dont la prime est entièrement prise en charge par leur employeur.

Une première remarque que l'on peut faire à ce stade est que la proportion d'individus possédant un contrat d'assurance décès dans l'échantillon est importante $(50.1 \%)$. La proportion relativement importante des assurés dans l'échantillon peut s'expliquer par l'âge des individus concernés par l'étude. On peut quand même noter qu'en France, ce type de contrats est peu représenté, au profit des contrats d'assurance vie-capitalisation et d'assurance en cas de vie. Toujours en considérant cette variable, on constate dans les résultats de l'enquête que les employeurs souscrivent souvent des contrats au profit de leurs employés $(28.9 \%$ ) ce qui s'explique peut être par l'insuffisance du système de protection sociale.

5 En France, l'appellation assurance vie regroupe un ensemble beaucoup plus grand de contrats, comprenant aussi les rentes viagères (assurance en cas de vie) et les contrats d'épargne disponible pour le souscripteur ou ses héritiers après une période donnée (assurance vie-capitalisation).

6 Ce chiffre est donné dans Cawley-Philipson (1999) qui se réfèrent aux statistiques données par le Conseil Américain d'Assurance Vle (American Council of Life Insurance). 
Les individus qui possèdent des contrats de groupe sont éliminés de notre étude car la prime par franc assuré qui correspond à ces contrats est liée à la probabilité moyenne de décès dans le groupe de souscripteurs et, pour ce qui est de ceux dont la prime est payée par l'employeur, il est impossible de savoir ce que l'individu aurait choisi si un contrat gratuit ne lui était pas proposé. Cette élimination, ainsi que celle des individus ne connaissant pas le type de contrat qu'ils possèdent, ramène le nombre d'individus dans notre échantillon à 5070 .

Les statistiques descriptives des variables utilisées, autres que celle concernant l'état de santé, sont reportées dans les deux tableaux suivants où le premier contient les variables numériques et le second, les variables qualitatives ${ }^{7}$.

\begin{tabular}{|l|c|c|c|c|c|}
\hline Variable & Nbre d'observations & Moyenne & Ecart type & Min & Max \\
\hline Àge (AGE) & 5070 & 55.60 & 3.98 & 45 & 65 \\
\hline Revenu annuel en \$ (REV) & 5070 & 47394.01 & 45711.06 & 75 & 600000 \\
\hline Prime annuelle en \$ (PRIME) & 1285 & 627.4 & 951.9 & 5 & 10800 \\
\hline Capital décès en \$ (CAP) & 1285 & 67022.4 & 110797.00 & 250 & 1250000 \\
\hline Prime/\$ assuré PRUN=PRIME/CAP & 1285 & 0.034 & 0.072 & 0.0002 & 0.988 \\
\hline Survie ₹75 ans (CROY) & 5070 & 6.26 & 3.08 & 0 & 10 \\
\hline
\end{tabular}

Tableau 1 : Statistiques descriptives : variables numériques

Le revenu annuel du ménage inclut le revenu des activités professionnelles : salaires, honoraires..., les retraites, ainsi que des intérêts, bénéfices ou remboursements de dettes...

\begin{tabular}{|c|c|c|}
\hline Variable & Effectif & Pourcentage \\
\hline sexe (SEXE) $\begin{array}{l}\text { femme } 0 \\
\text { homme } 1\end{array}$ & $\begin{array}{l}2477 \\
2593 \\
\end{array}$ & $\begin{array}{l}48.9 \\
51.1\end{array}$ \\
\hline $\begin{array}{l}\text { st. matrimonial (STM) } \\
\text { mariélibataire } 0 \\
\text { mare }\end{array}$ & $\begin{array}{l}1740 \\
3330 \\
\end{array}$ & $\begin{array}{l}34.3 \\
65.7\end{array}$ \\
\hline enfants (ENF) $\begin{array}{l}0 \\
1\end{array}$ & $\begin{array}{l}2257 \\
2813 \\
\end{array}$ & $\begin{array}{l}44.5 \\
55.5\end{array}$ \\
\hline contrat d'assurance décès (CONT) ${ }_{1}^{0}$ & $\begin{array}{l}3409 \\
1661\end{array}$ & $\begin{array}{l}67.2 \\
32.8\end{array}$ \\
\hline
\end{tabular}

Tableau 2 : Statistiques descriptives : variables qualitatives

Le statut matrimonial est ici considéré au sens large : les individus vivant en couple sont aussi considérés comme mariés.

7 Les analyses statistiques de notre étude sont effectuées avec le logiciel SAS (version 6.11) et l'histogramme est réalisés avec Statgraphics (version 5.1.). 
Le nombre d'enfants d'un ménage n'apparaît pas dans les données, la présence d'un enfant au moins étant le résultat du croisement de deux variables (présence d'enfants habitant dans le foyer, ou présence d'enfants habitant hors du foyer).

La différence entre le nombre d'individus possédant un contrat et le nombre d'individus dont la prime et le capital sont reportés, est due à des valeurs manquantes qui correspondent à des individus qui ne connaissent pas la prime qu'ils payent ni le montant du capital assuré.

Avant de tester le lien entre l'achat d'un contrat, le capital assuré et les caractéristiques des agents, nous effectuons une analyse dont l'objet est de déterminer dans quelle mesure les croyances des individus sont liées à leurs caractéristiques objectives, ici représentées par l'âge, le sexe et l'état de santé.

\section{Les croyances de survie : « optimisme », « pessimisme » ou « réalisme»}

Dans cette section, nous tentons d'isoler la partie objective des croyances de survie, à l'aide de variables corrélées avec la probabilité de survie. Avant d'entreprendre cette décomposition des croyances en partie objective et subjective, il nous paraît utile de considérer de plus près cette variable ${ }^{8}$, dont l'histogramme est représenté dans la figure 1 . Une analyse de la perception des risques, à partir de la même base de données, est faite par Hurd, McGarry (1993) : cependant, ces auteurs ne font pas ce type de décomposition et s'interressent principalement à la partie subjective des croyances.

On constate que $55.9 \%$ des individus estiment avoir des chances strictement supérieures à $50 \%$ de vivre au delà de 75 ans, alors qu'ils ne sont que $21.8 \%$ à penser que leurs chances sont strictement inférieures à $50 \%$. Ces chiffres laissent penser que les individus interrogés savent que la durée de vie moyenne correspondant à leur tranche d'âge est supérieure à 75 ans $^{9}$.

On remarque, par ailleurs, des pics d'effectifs aux extrémités de l'intervalle pour les croyances et au milieu : $8 \%$ des individus estiment qu'ils ne vivront pas au delà de 75 ans, $21 \%$, qu'ils vivrons sûrement et $22.3 \%$ estiment qu'ils ont $50 \%$ de chances de survie au delà de cette limite d'âge. On peut supposer que ceci est lié au fait que les individus ont une mauvaise perception des probabilités intermédiaires : ils sont plus prêts à considérer qu'un événement est impossible, certain ou a $50 \%$ de chances de se produire, que de donner une probabilité précise entre ces trois valeurs.

B Rappelons que cette variable contlent les réponses à la question : « En utillsant un nombre entre 0 et 10 où 0 signifie aucune chance et 10, la certitude, dites à combien vous estimez vos chances de vivre au delà de 75 ans?".

9 On peut mentionner que l'espérance de vie des américains qui avaient 45 ans en 1981 était de 74.6 ans pour les hommes et 80.6 ans pour les femmes d'aprés Hamermesh (1985) (les données proviennent de Vital Statistics of the United States). 


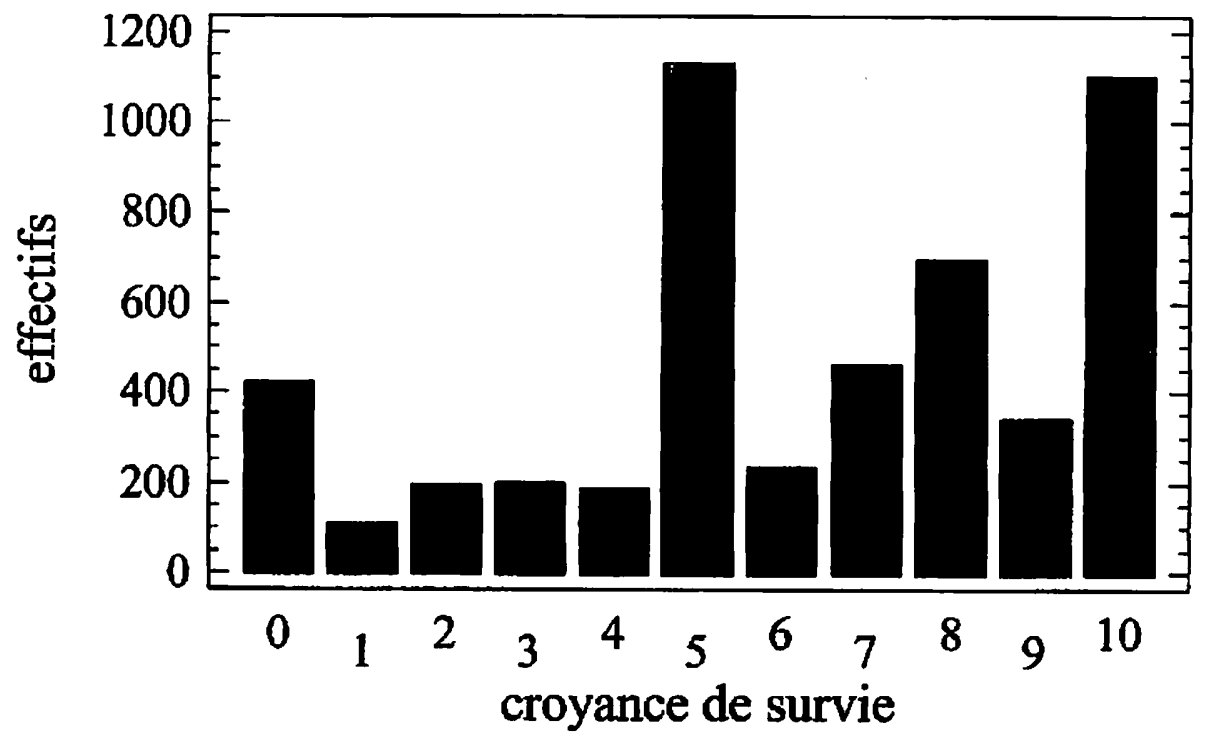

Figure 1 : Histogramme des croyances de survie au delà de 75 ans

Les chances qu'un individu estime avoir de vivre au delà d'un certain âge sont déterminées, à notre sens, par deux facteurs : l'un objectif, qui est directement lié à l'âge et à la présence ou l'absence de maladies pouvant avoir des conséquences graves et l'autre, subjectif, qui est lié à l'attitude vis-à-vis du risque de l'individu. A titre d'exemple, considérons trois individus, deux sont en bonne santé et le troisième souffre d'un cancer. Si l'individu souffrant du cancer, estime avoir moins de chances de vivre au delà de 75 ans que les individus bien portants, c'est pour une raison objective. En revanche, si l'un des individus bien portants juge ses chances inférieures à celles de l'autre c'est du pessimisme, puisque son état de santé est bon :il considère alors qu'un accident peut lui arriver.

Pour dégager les facteurs objectifs influents sur les croyances de survie, nous utilisons des variables relatives à l'état de santé et l'âge et nous estimons un modèle dans lequel les croyances sont expliquées par ces variables. Le résidu représente dans ce cas la partie subjective de la croyance.

La base de données utilisée incluant un questionnaire très complet sur l'état de santé, nous avons choisi, à partir d'un arbre de classification, les 8 variables les plus pertinentes pour l'explication des croyances.

Les variables sont codées 1 si l'individu possède le symptôme correspondant et 0 sinon $^{10}$.

Nous modélisons les croyances en fonction des variables de santé, de l'âge et du sexe. Les variables qualitatives étant dichotomiques, le modèle

10 La variable Hospitalisation a une valeur de 1 si lindividu interrogé a passé au moins une nuit à l'hôpital pendant l'année qui précède l'interview. 


\begin{tabular}{|c|c|c|}
\hline Variable & Effectif & Pourcentage \\
\hline Fumeur (FUM) ${ }_{1}^{0}$ & $\begin{array}{l}3606 \\
1464 \\
\end{array}$ & $\begin{array}{l}71.1 \\
28.9 \\
\end{array}$ \\
\hline Hypertension (HYP) ${ }_{1}^{0}$ & $\begin{array}{l}3744 \\
1326 \\
\end{array}$ & $\begin{array}{l}73.8 \\
26.2\end{array}$ \\
\hline Diabète (DIA) ${ }_{1}^{0}$ & $\begin{array}{c}4609 \\
461 \\
\end{array}$ & $\begin{array}{c}90.9 \\
9.1 \\
\end{array}$ \\
\hline Cancer (CAN) ${ }_{1}^{0}$ & $\begin{array}{c}4781 \\
289 \\
\end{array}$ & $\begin{array}{l}94.3 \\
5.7 \\
\end{array}$ \\
\hline Maladies cardio-vasculaires (CAR) $\begin{array}{l}0 \\
1\end{array}$ & $\begin{array}{l}4728 \\
342 \\
\end{array}$ & $\begin{array}{c}93.3 \\
6.7 \\
\end{array}$ \\
\hline Maladies gastro-entériologiques (GAS) ${ }_{1}^{0}$ & $\begin{array}{c}4548 \\
522 \\
\end{array}$ & $\begin{array}{l}89.7 \\
10.3 \\
\end{array}$ \\
\hline Cholestérol (CHO) ${ }_{1}^{0}$ & $\begin{array}{l}3855 \\
1215 \\
\end{array}$ & $\begin{array}{l}76.0 \\
24.0 \\
\end{array}$ \\
\hline Hospitalisation (HOS) $\begin{array}{l}0 \\
1\end{array}$ & $\begin{array}{l}4447 \\
623\end{array}$ & $\begin{array}{l}87.7 \\
12.3\end{array}$ \\
\hline
\end{tabular}

Tableau 3 : Statistiques descriptives : état de santé

linéaire général se ramène à une régression multiple avec des variables codées 0 ou 1 plus l'âge (variable numérique). Pour un individu $i$ :

$$
(\mathrm{CROY})_{i}=\mathrm{cste}+\sum_{j} \alpha_{j} X_{j i}+\eta_{i}
$$

où $X_{j}, j=1, \ldots, 10$ sont les variables de santé énumérées dans le tableau ci-dessus auxquelles sont ajoutées l'âge et le sexe (SEXE $=0$ si femme et SEXE $=1$ si homme) et $\alpha_{j}, j=1, \ldots, 10$ sont les paramètres inconnus à estimer. Les termes d'erreur $\eta_{i}$ suivent une loi $N(0, \sigma)$ pour tout $i$. Le modèle (11) est estimé par la méthode des MCO.

Les valeurs des coefficients estimés et les statistiques de Student sont reportées dans le tableau suivant:

\begin{tabular}{|c|c|c|c|c|c|c|c|c|c|c|c|}
\hline & FUM & HYP & DIA & CAN & CAR & GAS & CHO & HOS & AGE & SEXE & Cste \\
\hline coefficient & -0.62 & -0.51 & -1.01 & -0.63 & -1.46 & -0.90 & -0.32 & -0.51 & 0.02 & -0.21 & 6.22 \\
\hline$t$ & -6.74 & -5.22 & -6.80 & -3.50 & -8.34 & -6.51 & -3.22 & -3.84 & 1.56 & -2.45 & 10.57 \\
\hline
\end{tabular}

Le coefficient de détermination et l'écart-type résiduel ont les valeurs suivantes : $R^{2}=0.076, \quad \hat{\sigma}=2.96$.

Malgré un $R^{2}$ assez faible, ce modèle met en évidence la rationalité, en moyenne, des personnes interrogées.

- Les maladies et le fait de fumer, ont un effet négatif sur la croyance de dépasser 75 ans.

Les maladies qui ont le poids le plus fort dans la réduction des croyances de survie sont les maladies cardio-vasculaires et le diabète : ce fait s'explique par la tranche d'âge dans laquelle se trouvent les personnes interrogées. Parmi les causes de mortalité non accidentelles correspondant à cette 
tranche d'âge, les attaques cardiaques et les infarctus tiennent la première place.

- Les hommes ont des croyances de survie, toutes choses égales par ailleurs, inférieures à celles des femmes.

Les individus semblent donc conscients du fait que les femmes ont une durée de vie supérieure à celle des hommes.

- L'âge n'est pas une variable significative.

Ce résultat peut paraître surprenant, dans la mesure où, statistiquement, la probabilité de survie au delà d'un certain âge est croissante par rapport à l'âge déjà atteint. La non significativité de cette variable dans la formation des croyances peut provenir de deux effets contradictoires : la réalité statistique évoquée ci-dessus, qui doit être prise en compte par certains individus et une dimension psychologique qui incite les individus âgés à considérer «qu'ils n'en ont plus pour très longtemps », indépendamment de leur état de santé.

L'élimination de l'âge du modèle ne modifie quasiment pas $R^{2}$, ni $\hat{\sigma}$.

On a donc une confirmation de l'hypothèse du modèle théorique sur le lien entre les croyances et certains déterminants du risque objectif.

Cette analyse nous permet de décomposer les croyances en deux parties : la partie expliquée par les caractéristiques objectives (qui correspond à $1-\tilde{p}$ dans le modèle théorique, puisque $\tilde{p}$ mesure la probabilité de décès) et la partie subjective qui provient de facteurs psychologiques et qui peut refléter l'attitude vis-à-vis de l'incertitude des agents (correspond à $-\varepsilon$ dans le modèle théorique). Pour un individu, la croyance objective sera représentée par la valeur prédite de la régression (11). La croyance subjective sera représentée par l'erreur de prédiction. On aura donc, pour chaque individu $i$ :

$$
(\mathrm{CROY})_{i}=(\mathrm{COBJ})_{i}+(\mathrm{CSUB})_{i}
$$

$\operatorname{avec}(\mathrm{COBJ})_{i}=\widehat{C s t e}+\sum_{j} \hat{\alpha}_{j} X_{j i}$ et $(\mathrm{CSUB})_{i}=\eta_{i}$.

Les principales caractéristiques de la distribution des croyances subjectives sont données ci-dessous, la figure 2 représente son histogramme des effectifs.

\begin{tabular}{|c|c|c|c|c|}
\hline & Moyenne & Écart type & Min & Max \\
\hline CSUB & 0 & 2.96 & -7.13 & 7.23 \\
\hline
\end{tabular}

On constate que cette distribution est très étalée ce qui montre que, pour un certain nombre d'individus, les caractéristiques objectives ne suffisent pas pour expliquer les croyances et qu'il existe un important élément subjectif. Le signe de la partie subjective des croyances correspond ici à l'attitude des individus vis-à-vis de l'incertitude. En rappelant que les croyances donnent les chances de survie et non de décès, un résidu positif signifie que 


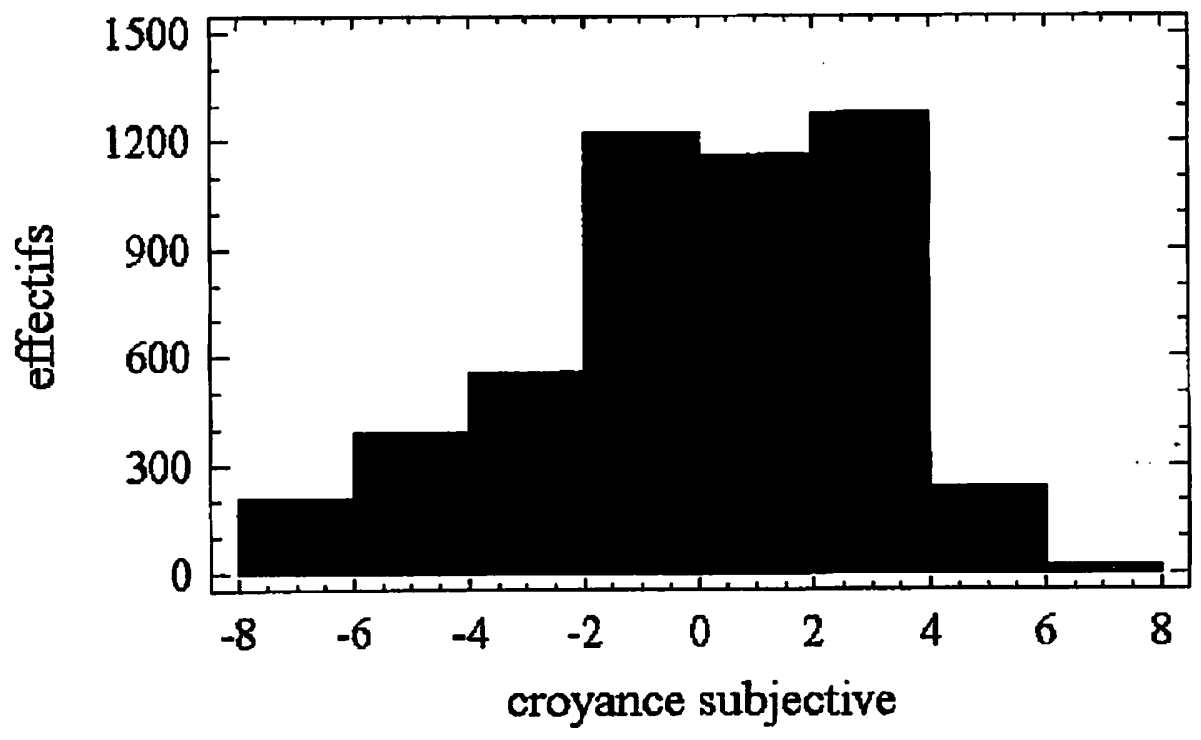

Figure 2 : Histogramme des croyances subjectives

l'individu surestime sa probabilité de survie, et donc sous-estime sa probabilité de décès; si le résidu est négatif, l'individu sous-estime sa probabilité de survie et donc surestime sa probabilité de décès. $53.3 \%$ des individus interrogés ont des croyances subjectives positives. Dans cette catégorie, il existe aussi des individus dont les croyances subjectives sont proches de 0 et qui font partie de la catégorie que nous appelons « les réalistes ». Si on ne considère que les croyances qui sont en dehors de l'intervalle $[-2,2]$, on peut dire que $29.6 \%$ des individus sont pessimistes et $30.3 \%$ optimistes. Les réalistes représentent alors $40.1 \%$ des individus interrogés.

Les résultats obtenus dans cette section peuvent être comparés à ceux de Hamermesh (1985), qui étudie, sur un échantillon d'économistes, les déterminants des croyances de survie. Les variables explicatives des croyances sont la présence de parents et grands parents âgés, le faite de fumer, de faire du sport et de souffrir de maladies qui peuvent avoir une issue fatale. L'auteur constate que le fait d'avoir des parents âgés et des grands parents qui ont atteint un âge avancé accroit les croyances de survie. Par ailleurs, le fait de fumer et de souffrir de maladies graves a l'effet inverse sur les croyances de survie. Nos résultats vont dans l'ensemble dans le même sens, cependant le fait d'avoir des informations plus détaillées sur l'état de santé permet une analyse plus fine du poids des différentes maladies dans la formation des croyances.

Dans les données d'enquête qu'utilise Hamermesh on demande aux individus une estimation de leur durée de vie, ce qui permet de comparer leurs estimations avec les données des tables de mortalité. L'auteur constate que ces croyances sont en moyenne proches des données actuarielles (durées 
de vie figurant dans les tables de mortalité), mais que leur variance est élevée. Ce résultat donne une justification supplémentaire à l'utilisation de la partie objective des croyances comme estimation du risque objectif des individus.

\section{$5 \quad$ La demande d'assurance décès}

Notre objectif dans cette section est de dégager les variables qui expliquent la demande d'assurance décès et de tester les prédictions des modèles théoriques, aussi bien sur la pertinence des variables qu'ils font intervenir, que sur leur effet sur la demande d'assurance. Étant donné que l'assurance décès est, sauf dans des cas spécifiques (l'achat d'un contrat d'assurance décès peut être demandé comme garantie du remboursement d'un emprunt), un contrat d'assurance non obligatoire, les résultats de cette analyse peuvent avoir des implications pour les stratégies de distribution des produits d'assurance vie, notamment dans la détermination de la population cible.

La décision d'assurance a deux composantes : les individus doivent dé cider à la fois s'ils s'assurent ou non et choisir le montant du capital assuré (qui correspond ici à la qualité de la couverture). La variable qu'on cherche à expliquer est alors le capital assuré. Le modèle que nous utilisons est un tobit généralisé (Gouriéroux 1989) qui permet de modéliser simultanément la décision de s'assurer ou non, et si oui le montant du capital assuré (en logarithme). En effet, le montant du capital est une variable tronquée, résultant d'une décision prise par les individus.

On suppose tout d'abord que le comportement observé $y_{i}=1$ si achat, $y_{i}=0$ sinon, pour un individu $i$ résulte d'une première variable latente $z$, inobservable et continue, combinaison linéaire des variables exogènes qui sont ici la partie objective et subjective des croyances des individus, leur âge et revenu, ainsi que les paramètres liés à l'altruisme : le statut matrimonial et la présence d'enfants :

$$
z_{i}=x_{i} \beta^{(1)}+u_{i 1}
$$

Lorsque $z$ reste inférieure à un certain seuil, on observe que l'individu n'achète pas d'assurance et, lorsqu'elle dépasse ce seuil, on observe l'achat d'assurance. Ainsi :

$$
\begin{aligned}
& y_{i}=1 \text { si } z_{i} \geqslant 0 \\
& y_{i}=0 \text { si } z_{i}<0
\end{aligned}
$$

Cette indicatrice $y_{i}$ nous indique s'il est possible ou non d'observer le montant de capital assuré, représenté par une seconde variable latente $r^{*}$, expliquée ici par les mêmes ${ }^{11}$ variables exogènes car nous souhaitons entre

11 Dans le modèle tobit généralisé, ces deux ensembles de variables ne sont pas nécessairement identiques. 
autres déterminer si les deux décisions (décisions d'assurance et montant du capital assuré) dépendent des mêmes facteurs ou non :

$$
r_{i}^{*}=x_{i} \beta^{(2)}+u_{i 2}
$$

et telle que le montant observé $r_{i}=\ln (C A P)_{i}$ est donné par :

$$
\begin{aligned}
& r_{i}=r_{i}^{*} \text { si } z_{i} \geqslant 0 ; \\
& r_{i}=\emptyset \text { si } z_{i}<0
\end{aligned}
$$

$r_{i}=\emptyset$ désignant l'absence d'observation (ce qui est différent d'une valeur nulle).

On suppose ici que les erreurs $u_{i 1}$ et $u_{i 2}$ sont normales. $r^{*}$ et $z$ sont en général corrélées car les décisions concernant l'achat ou non d'assurance et la qualité de la couverture sont liées. L'estimation des paramètres $\beta^{(1)}$ et $\beta^{(2)}$ s'effectue par la méthode du maximum de vraisemblance

$$
\begin{aligned}
z_{i}= & \left.\beta_{0}^{(1)}+\beta_{1}^{(1)}(\mathrm{STM})_{i}+\beta_{2}^{(1)}(\mathrm{ENF})_{i}+\beta_{3}^{(1)}(\mathrm{CSUB})_{i}+\beta_{4}^{(1)}(\mathrm{COBJ})_{i} 14\right) \\
& +\beta_{5}^{(1)}(\mathrm{AGE})_{i}+\beta_{6}^{(1)}(\mathrm{REV})_{i}+u_{i 1}
\end{aligned}
$$

Le tableau suivant donne les paramètres estimés, ainsi que leurs erreurs standard. NS veut dire non significatif au seuil $5 \%$.

\begin{tabular}{|c|c|c|c|c|c|c|c|}
\hline & Cste & STM & ENF & CSUB & COBJ & AGE & REV \\
\hline$\hat{\beta}^{(1)}$ & -0.246 & 0.077 & 0.14 & -0.009 & -0.01 & -0.005 & $2.045 * 10^{-6}$ \\
\hline erreur standard & 0.278 & 0.040 & 0.037 & 0.005 & 0.500 & 0.004 & $0.37 * 10^{-6}$ \\
\hline & & NS & & NS & NS & NS & \\
\hline
\end{tabular}

On constate que les seules variables qui sont significatives dans la décision d'acheter ou non un contrat d'assurance décès sont le fait d'avoir des enfants et le revenu.

- L'accroissement du revenu a un effet positif sur la probabilité qu'un individu achète un contrat d'assurance décès.

Ce résultat laisse supposer que l'assurance décès est un bien normal, dont la consommation augmente lorsque le revenu augmente, ce fait ne peut être complètement établi qu'une fois qu'on aura étudié l'impact de la richesse sur le capital assuré.

- Le fait d'avoir des enfants a un effet positif sur la probabilité de s'assurer.

On a donc une confirmation du fait que la présence d'héritiers est déterminante pour la décision de s'assurer : le paramètre d'altruisme, présent dans le modèle théorique se trouve ainsi justifié.

- Les croyances n'ont pas d'effet sur la décision d'acheter un contrat d'assurance décès. 
- L'âge n'est pas significatif ici, ce qui peut s'expliquer par les résultats d'Arrondel, Masson (1997) qui étudient la demande d'assurance décès en fonction de l'âge et montrent que aux alentours de la tranche d'âge considérée ici (45-65 ans), la demande d'assurance décès augmente, puis diminue avec l'âge.

On déduit de cette analyse que le risque n'a pas d'effet sur l'achat ou le non achat d'un contrat d'assurance décès. Les seuls paramètres qui interviennent sont la présence d'héritiers et la richesse. En considérant que le paramètre d'altruisme est nul lorsque l'individu n'a pas d'héritiers, on a une confirmation empirique du résultat (i) de la Proposition 1.

Les coefficients de la deuxième équation du tobit sont :

\begin{tabular}{|c|c|c|c|c|c|c|c|}
\hline & Cste & STM & ENF & CSUB & COBJ & AGE & REV \\
\hline$\hat{\beta}^{(2)}$ & 11.33 & 0.055 & 0.933 & -0.053 & -0.192 & -0.065 & $1.071 * 10^{-5}$ \\
\hline erreur standard & 0.54 & 0.082 & 0.076 & 0.011 & 0.04 & 0.0082 & $7.1 * 10^{-7}$ \\
\hline & & NS & & & & & \\
\hline
\end{tabular}

où

$$
\begin{aligned}
r_{i}^{*}= & \ln (C A P)_{i}=\beta_{0}^{(2)}+\beta_{1}^{(2)}(\mathrm{STM})_{i}+\beta_{2}^{(2)}(\mathrm{ENF})_{i}+\beta_{3}^{(2)}(\mathrm{CSUB})_{i} \\
& +\beta_{4}^{(2)}(\mathrm{COBJ})_{i}+\beta_{5}^{(2)}(\mathrm{AGE})_{i}+\beta_{6}^{(2)}(\mathrm{REV})_{i}+u_{i 2}
\end{aligned}
$$

Les principaux résultats de cette estimation sont ainsi les suivants :

- Les variables significatives pour l'explication du capital décès diffèrent de celles qui expliquent l'achat d'un contrat.

Les croyances objectives et subjectives, ainsi que l'âge, sont les variables qui ont un impact sur le capital décès acheté sans intervenir dans la décision d'achat d'un tel contrat.

- Le revenu a un effet positif et significatif sur le capital décès.

On a une confirmation du fait, établi dans le paragraphe précédent, que l'assurance décès est un bien normal. L'accroissement de la richesse, toutes choses égales par ailleurs, entraîne un accroissement du capital décès : l'effet substitution, qui consiste à accroître l'épargne lorsque le revenu augmente, est donc inférieur à l'effet richesse.

- Les croyances de survie, objectives et subjectives ont un effet négatif sur le capital décès.

Avant de comparer ce résultat avec ceux du modèle théorique, rappelons que dans ce dernier les croyances considérées étaient les croyances de décès et non de survie.

Dans le modèle théorique, l'effet des croyances de décès sur le capital assuré est positif, sans hypothèses supplémentaires sur la fonction d'utilité soit lorsque c'est la partie subjective des croyances qui augmente, soit lorsque les croyances et la prime unitaire sont indépendantes. Les résultats 
empiriques que nous obtenons vont dans le sens de la seconde hypothèse, car, non seulement les deux parties des croyances de survie ont un effet négatif sur le capital, mais l'amplitude de ces effets diffèrent : ce sont les croyances objectives qui semblent influencer plus fortement le montant du capital décès (rappelons que l'amplitude relative des deux effets est indéterminée dans le modèle théorique).

- L'âge a un effet négatif sur le capital assuré.

Nous avons constaté, lors de l'étude de l'impact des caractéristiques objectives sur les croyances, que ces dernières n'étaient pas liées à l'âge des individus. Ainsi, ce n'est pas le risque qui explique la réduction du capital assuré avec l'âge. Cet effet peut trouver une justification du côté de l'autre motif d'achat d'assurance décès, qui est l'altruisme. Deux effets peuvent intervenir : un individu qui achète de l'assurance décès pour assurer l'avenir de ces enfants, en achètera pour un montant d'autant plus élevé que ses enfants sont jeunes. En revanche, lorsque les enfants sont plus âgés et peuvent se prendre en charge, situation plus probable lorsque l'individu est plus âgé, le capital décès pourra être plus réduit. Par ailleurs, on peut supposer qu'un individu qui se rapproche de la période de la retraite, anticipe une baisse proche de son revenu et donc sera incité à épargner plus.

Pour résumer, les résultats des analyses sur la demande d'assurance décès que nous avons effectuées montrent d'abord que l'achat ou non d'assurance décès et le montant de capital assuré ne semblent pas être des décisions jointes, mais plutôt séparées : si la décision d'acheter de l'assurance est liée uniquement au revenu et au fait d'avoir des enfants, dans la détermination du capital décès apparaissent des variables liées au risque de décès objectif des individus et à l'attitude vis-à-vis de ce risque.

L'effet des variables liées à l'altruisme est bien en accord avec le modèle théorique, il en est de même pour la partie subjective des croyances. Pour ce qui est de la partie objective (liée au risque réel), son impact était indéterminé ${ }^{12}$ dans le modèle théorique et ici il apparaît conforme à l'intuition.

L'impact de la richesse montre que l'assurance décès est un bien normal pour la plupart des individus interrogés.

\section{Conclusion}

L'étude empirique menée dans cet article avait comme principal objectif de tester l'impact des croyances de survie sur la demande d'assurance décès. Elle nous a donné par ailleurs l'occasion, d'une part d'analyser la formation

12 Remarquons que dans le modèle théorique, nous avons supposé que si les croyances sont lièes au risque objectif, la prime unitaire l'est aussi ce qui n'est pas établi lci. 
des croyances de survie et, d'autre part, de déterminer l'impact d'autres paramètres sur la décision d'achat d'un tel contrat.

La volonté de trouver une base de données où apparaîssent simultanément la perception d'un risque par les individus et leurs décisions d'assurance de ce risque nous a amenée à effectuer cette étude dans le cadre de l'assurance décès, qui est un contrat de type particulier à plusieurs titres. Il serait aussi intéressant d'effectuer le même type d'analyse sur des données d'assurance-dommage car dans ce cadre il est possible de mettre en évidence les comportements que seule une différence de perception des risques entre assureurs et assuré peut expliquer. Par ailleurs, lorsqu'un seul risque est considéré, il est impossible de différencier les comportements issus d'une perception des risques qui correspond à une probabilité subjective, de ceux issus d'une perception qui correspond à un modèle plus général d'espérance d'utilité. Pour effectuer cette distinction, il semble donc nécessaire de considérer plusieurs risques.

\section{References}

Arrondel, L. et A. Masson (1997), «L'assurance vie est-elle une épargne de luxe? ", Risques, 29, pp. 125-154.

Benjamin D. et W. Dougan (1997), "Individuals' Estimates of the Risks of Death : Part 1 - A Reassessment of the Previous Evidence", Journal of Risk and Uncertainty, 15, pp. 115-133.

Cawley, J. et T. Philipson (1999), "An Empirical Examination of Information Barriers to Trade in Insurance", American Economic Review, Vol. 89, 4, pp. 827-848.

Cohen M. et J. M. TALLON (2000), Décision dans le risque et l'incertain : l'apport des modèles non-additifs, Revue d'Économie Politique, Bilans et Essais, 5, pp. 631-681.

Eeckoudt L. et C. Gollier (1992), Les Risques Financiers : Évaluation, Gestion, Partage, Ediscience.

Fisher, S. (1973), "A life cycle model of life insurance purchases", International Economic Review, 14, pp. 132-152.

Gourieroux, C. (1989), Économetrie des variables qualitatives, Economica, Paris.

Henriet, D. et J. C. Rochet (1992), Microéconomie de l'assurance, Economica, Paris.

Hamermesh, D.S (1985), "Expectations, Life Expectancy, and Economic Behavior", Quarterly Journal of Economics, May, pp. 289-408.

Hurd, M. et K. McGarry (1993), "Evaluation of Subjective Probability Distributions in the HRS", NBER Working Paper n. 4560. 
INRETS (1994), «Liens entre la surestimation de ses propres capacités, l'expérience de la conduite et l'activité de conduite. ", rapport N. 187.

Lichtenstein, S., Slovic P., Fischoff B. et alii (1978), "Judged Frequency of Lethal Events", Journal of Experimental Psychology, 4, pp. 551-578.

Lusardi, A. (1998), "On the Importance of the Precautionary Saving Motive", American Economic Review, Vol. 88, 2, pp. 449-453.

Mitchell, O.S. et J. F. Moore (1998), "Retirement Wealth Accumulation and Decumulation : New Developments and Outstanding Opportunities", Journal of Risk and Insurance, Vol. 65, 3, pp. 371-400.

Rogowski, J. et L. Karoly (2000), "Health Insurance and Retirement Behavior : Evidence from the Health and Retirement Survey", Journal of Health Economics, Vol. 19, 4, pp. 529-539.

Schlesinger, H. (2000), "The theory of insurance demand", dans Handbook of Insurance, ed. G. Dionne, Kluwer Academic Publishers.

Viscusi, K. (1990), "Do Smokers Underestimate Risks ?", Journal of Political Economy, vol. 98 (6), pp. 1253-1269.

Viscusi, K., Hakes J. et A. CARLIN (1997), "Measures of Mortality Risks", Journal of Risk and Uncertainty, 14, pp. 213-233.

Willinger, M. (1990), «La rénovation des fondements de l'utilité et du risque », Revue Économique, 41, pp. 5-48.

Yaari, M. (1965), "Uncertain lifetime, life insurance and the theory of the consumer", Review of Economic studies, pp. 137-150. 